\title{
Correlation between the prevalence of certain fungi and sick building syndrome
}

\author{
J Danny Cooley, Wing C Wong, Cynthia A Jumper, David C Straus
}

\begin{abstract}
Objective-To examine the role of fungi in the production of sick building syndrome. Methods-A 22 month study in the United States of 48 schools (in which there had been concerns about health and indoor air quality (IAQ)). Building indoor air and surface samples, as well as outdoor air samples were taken at all sites to look for the presence of fungi or their viable propagules.
\end{abstract}

Results-Five fungal genera were consistently found in the outdoor air and comprised over $95 \%$ of the outdoor fungi. These genera were Cladosporium (81.5\%), Penicillium (5.2\%), Chrysosporium (4.9\%), Alternaria (2.8\%), and Aspergillus (1.1\%). At 20 schools, there were significantly more colony forming units per cubic metre $\left(\mathrm{CFU} / \mathrm{m}^{3}\right) \quad(\mathrm{p}<0.0001)$ of propagules of Penicillium species in the air samples from complaint areas when compared with the outdoor air samples and the indoor air samples from noncomplaint areas. At five schools, there were more, although not significant $(p=0.10)$, Penicillium propagules in the air samples from complaint areas when compared with the outdoor air samples and the indoor air samples from noncomplaint areas. In 11 schools, the indoor air (complaint areas) fungal ratios were similar to that in the outdoor air. In these 11 schools Stachybotrys atra was isolated from swab samples of visible growth under wetted carpets, on wetted walls, or behind vinyl wall coverings. In the remaining 11 schools, the fungal ratios and $\mathrm{CFU} / \mathrm{m}^{3}$ of air were not significantly different in different areas. Many of the schools took remedial action that resulted in an indoor air fungal profile that was similar to that outdoors.

Conclusions-Propagules of Penicillium and Stachybotrys species may be associated with sick building syndrome.

(Occup Environ Med 1998;55:579-584)

Keywords: fungi; sick building syndrome; indoor air quality; spores

Reports about buildings with air related problems have appeared increasingly often after the early 1970s, although this problem has certainly been with humans for centuries. ${ }^{12}$ Sick building syndrome (SBS), a commonly used term for symptoms resulting from problems with indoor air quality (IAQ), was first recognised as an important problem affecting people in certain buildings in 1982. The first official study of SBS that examined more than one structure was published in $1984 .^{3}$ Sick building syndrome has been difficult to define and no single cause of this malady has been identified. ${ }^{1}$

Complaints common to SBS include allergic rhinitis, difficulty in breathing, headaches, flulike symptoms, and watering of the eyes. ${ }^{4}$ Numerous studies have been carried out in an attempt to elucidate the cause of SBS. ${ }^{35}$ Early studies showed that many of the reported causes of SBS were undesirably high levels of known respiratory irritants such as nitrogen and sulphur dioxides, hydrocarbons, and particulates, ${ }^{6}$ known or suspected carcinogens such as asbestos, radon, formaldehyde, and tobacco smoke, ${ }^{7}$ or chemicals being released by new building materials.

Although fungal spores are universal atmospheric components both indoors and outdoors and are now generally recognised as important causes of respiratory allergies, ${ }^{8-13}$ there are few studies showing which fungi and spores are associated with IAQ problems. ${ }^{14}$ This study was made possible due to our association with an IAQ company. The uniqueness of this study was that the sites were made available because the school officials contacted the IAQ company. This allowed us access to all the samples, data, questionnaires, and occupant generated complaints from schools that were experiencing IAQ problems.

Although no one cause for the symptoms induced by IAQ problems is likely to exist, the presence of fungi in sick buildings is becoming consistently associated with this problem. ${ }^{4}{ }^{15-17}$ Fungal contamination in indoor environments has been shown to produce allergies in occupants of these buildings. ${ }^{18}{ }^{19}$ Even though the IAQ company's investigations were broadly based and in depth, the presence of fungi was the primary focus of this study, in which we present evidence for the role of Penicillium species and Stachybotrys species in buildings with IAQ problems.

\section{Materials and methods}

SURVEY PROCEDURES

The 22 month study examined 48 schools that were experiencing IAQ problems. These were in states along the United States Gulf of Mexico and the Atlantic seaboard. The sites were surveyed with the following criteria: collection of building characterisation data based on direct inspection and interviews with building occupants; building characterisation including measurement of temperature and humidity, examination of heating, ventilation, 
and air conditioning (HVAC) systems and physical examination of the building; particulates, $\mathrm{CO}_{2}$, and chemical measurements; inspection of sites that had been wetted or showed mould growth; swab samples and air samples taken by an Andersen air sampler; and administration of a questionnaire to building occupants, or access to occupant generated complaints (letters to school officials) and other complaint surveys conducted by other agencies - such as public health departments.

The questionnaire that was administered by the IAQ company was designed to determine the areas or rooms in which the occupants had complaints about the IAQ. The questionnaire asked for such information as the nature of the complaint, symptom patterns, timing patterns, and observations about building conditions that might explain the symptoms found. The questionnaire's answers and comments, along with the occupant generated complaints and surveys from other agencies, were placed into the following categories: (a) type of symptoms; (b) when do the symptoms start; (c) when do the symptoms go away; (d) when are the symptoms worst; (e); pre-existing symptoms (allergies, asthma, etc); $(f)$ discomfort complaints (noise, temperature, odours, etc); and ( $g$ ) complaint areas (rooms).

Non-complaint areas were also identified. The minimum criteria for designation as a non-complaint area were (a) all occupants, whose primary location was the non-complaint area, had no IAQ complaints, and (b) the HVAC system was separate from any complaint areas.

\section{MICROBIOLOGICAL IDENTIFICATION}

Air samples were taken with a two stage Bioaerosol Sampler (Model 2000 Andersen Samplers, Atlanta, GA, USA) at a calibrated flow rate of $28.4 \mathrm{l} / \mathrm{min}$ for 5 minutes. During sampling, the Andersen samplers were placed about $1 \mathrm{~m}$ above floor level. Sabouraud's Dextrose agar (SDA) pH 5.6 was used for air sampling and swab sampling. Plates were incubated at $22^{\circ} \mathrm{C}$ and $90 \%$ relative humidity $(\mathrm{RH})$ for up to 14 days. The isolated fungi were identified with standard identification techniques. ${ }^{20-23}$ Colony forming units per cubic metre $\left(\mathrm{CFU} / \mathrm{m}^{3}\right)$ of air were calculated with the formula:

$\mathrm{CFU} / \mathrm{m}^{3}=$ (number of CFU / ( minutes sampled $\left(1 \mathrm{ft}^{3} /\right.$ minute $\left.)\right)\left(35.3 \mathrm{ft}^{3} / \mathrm{m}^{3}\right)$

The total fungal $\mathrm{CFU} / \mathrm{m}^{3}$ for each air sample was calculated and the ratio for each organism per sample was determined. The results were entered according to the area that was sampled (the outdoor air sample areas, the indoor air samples complaint areas or the indoor air sample non-complaint areas) and the average $\mathrm{CFU} / \mathrm{m}^{3}$ and ratio, in terms of percentage, for each organism was determined for each area examined.

Using sterile swabs, samples were taken from areas of visible fungal growth, HVAC systems, wetted areas, standing water, dead air spaces, and areas of dust accumulation. The swabs were either placed into sterile plastic bags for transport to the laboratory or streaked undiluted on to agar $\mathrm{pH} 5.6$ plates. At the laboratory, the swab tip was placed into a sterile tube containing $10 \mathrm{ml}$ sterile phosphate buffered saline and vigorously vortexed for one minute. Samples $(100 \mu \mathrm{l})$ were pipetted on to agar plates $(\mathrm{pH} 5.6)$ and spread with sterile rods. The plates were incubated at $22^{\circ} \mathrm{C}$ and $90 \%$ relative humidity for up to 14 days. The fungi were identified and the fungal growth on the plates was estimated with the following criteria: $0 \mathrm{CFU}$, no growth; 1-5 CFU, very light growth; 6-10 CFU, light growth; 11-30 CFU, medium growth; 31-50 CFU, heavy growth; and $>50$ CFU, very heavy growth.

CARBON DIOXIDE MEASUREMENTS

The $\mathrm{CO}_{2}$ content of the air, expressed in parts per million (ppm), was monitored with a Ventostat $\mathrm{CO}_{2}$ Sensor (Model 1070/1071, Telaire Systems, Delspo, Sweden).

\section{CHEMICAL MEASUREMENTS}

An independent laboratory was contracted by the IAQ company to perform sampling for various indoor air components such as formaldehyde, nitrogen dioxide, hydrogen sulphide, sulphur dioxide, and carbon monoxide. Formaldehyde samples were collected with a Sensidyne/Gastec (Sensidyne/Gastec, Goleta, CA) collector in association with colorimetric indicator tubes with a lower detection limit of $0.1 \mathrm{ppm}$. Nitrogen dioxide, hydrogen sulphide, sulphur dioxide, and carbon monoxide samples were collected with a MultiRAE PGM-50 gas sampling device (Rae Systems, Sunnyvale, CA, USA). The collector was calibrated before sampling for each particular component. Outdoor and indoor samples were taken in the complaint and non-complaint areas. All sampling was performed in accordance to the manufacturers suggested use.

MEASUREMENTS OF PARTICULATES, TEMPERATURE, AND RELATIVE HUMIDITY Airborne particles were counted with an APC1000 Airborne Particle Counter (Biotest Diagnostics, Denville, NJ, USA). The APC-1000 detects particles relative to four thresholds: $>0.3,0.5,1.0$, and $5.0 \mu \mathrm{m}$. The APC-1000 also measures temperature and relative humidity.

REMEDIAL ACTION IN BUILDINGS

Any building materials that showed physical deterioration were removed and replaced. Existing microbial contamination on intact and structurally sound surfaces was cleaned and treated with an approved disinfectant. The remedial measures to the HVAC consisted of the removal of visible surface contaminants and the cleaning of air side surfaces of all internal air handling surfaces including, but not limited to, fans, coils, drain pans, filter racks, motors, dampers, and specific air ducts. Any damaged or delaminated insulation within the airducts being cleaned and sanitised was replaced. All work areas inside the air handlers, air ducts, and equipment rooms were isolated and kept under negative air pressure with high efficiency particulate arrestor filtered negative 
Incidences per 100 employees (95\% CI) of reported complaints and symptoms regarding indoor air quality (IAQ) at 48 United States schools between 1994 and 1996

\begin{tabular}{|c|c|c|c|c|c|c|c|c|}
\hline Type of symptom & Incidence & $95 \% C I$ & Type of symptom & Incidence & $95 \% C I$ & Type of symptom & Incidence & $95 \% C I$ \\
\hline Nasal drainage and congestion & 19.8 & \pm 1.3 & Discomfort complaints & & & When are symptoms the worst? & & \\
\hline Itchy or watering eyes & 14.3 & \pm 1.1 & Odorus & 5.2 & \pm 0.4 & High humidity & 12.0 & \pm 0.9 \\
\hline Contact problems & 5.6 & \pm 1.2 & Temperature (hot/cold) & 7.2 & \pm 0.1 & Low humidity & 0.0 & \pm 0.0 \\
\hline Headaches & 12.5 & \pm 0.6 & Noise & 0.8 & \pm 0.3 & Spring & 3.9 & \pm 0.8 \\
\hline Sinus & 10.3 & \pm 0.5 & Ventilation & 6.1 & \pm 0.3 & Summer & 0.0 & \pm 0.0 \\
\hline Severe & 3.4 & \pm 0.4 & & & & Fall & 2.7 & \pm 0.6 \\
\hline Increased airway infections & 14.3 & \pm 1.0 & Onset of symptoms & & & Winter & 4.5 & \pm 0.5 \\
\hline Cough & 6.5 & \pm 0.6 & Entering the building & 3.4 & \pm 0.9 & Start of school & 5.7 & \pm 2.0 \\
\hline Shortness of breath & 5.9 & \pm 0.4 & Working in the building & 11.0 & \pm 1.7 & Morning & 3.4 & \pm 0.4 \\
\hline Sneezing & 6.8 & \pm 1.0 & Start of school & 11.3 & \pm 1.9 & Afternoon & 1.1 & \pm 0.3 \\
\hline Dizziness & 2.2 & \pm 0.5 & & & & Monday & 0.8 & \pm 0.3 \\
\hline Fatigue & 1.1 & \pm 0.3 & When do symptoms go away? & & & Late in week & 0.8 & \pm 0.3 \\
\hline Flu-like symptoms & 1.8 & \pm 0.6 & Never & 3.5 & \pm 08 & No pattern & 1.1 & \pm 0.3 \\
\hline Nausea & 1.8 & \pm 3.4 & Leave work & 2.1 & \pm 0.6 & Always & 2.3 & \pm 0.6 \\
\hline Allergies & 17.0 & \pm 1.0 & Weekends & 4.3 & \pm 0.9 & Before remedy & & \\
\hline Asthma & 1.4 & \pm 0.3 & Vacations & 14.7 & \pm 2.5 & IAQ complaints or symptoms & 31.3 & \pm 6.8 \\
\hline \multirow[t]{2}{*}{ Other health conditions } & 1.2 & \pm 0.5 & Medications & 4.4 & \pm 0.2 & After remedy & & \\
\hline & & & & & & IAQ complaints or symptoms & 2.5 & \pm 1.1 \\
\hline
\end{tabular}

air machines to prevent migration of particulates. The cleaning was conducted in accordance with the National Air Duct Cleaners Association Standard 1992-01. ${ }^{24}$ All work was done after hours or at weekends. All personnel involved in the remedial work had the proper safety equipment and training and the Occupational Health and Safety Administration standards were observed. Air and swab samples were retaken within 60 days and after at least 6 months of completion of remedial work.

DATA ANALYSIS

Data were analysed by a computer program (Sigma Stat) with the Mann-Whitney rank sums test ( $U$ test), the Kruskal-Wallis one way analysis of varience (ANOVA) ( $H$ test), Spearman's product moment correlation, and Dunn's multiple comparisons and partial correlations. $^{25}$

\section{Results}

COMPLAINTS

Of the 48 schools surveyed, 40 were elementary schools (children aged 5-10). At most of these sites the school nurse distributed the IAQ company's questionnaire to only the staff. There were 622 occupants that reported IAQ symptoms or complaints, which represented $28 \%$ of the total staff (students were not included). With the exception of nausea, there were no significant differences between the reported complaints and symptoms at the different sites. All of the sites were combined and the average incidence per 100 employees along with the $95 \%$ confidence intervals $(95 \%$ CIs) are displayed in the table. Nasal drainage and congestion (IR $19.3,95 \% \mathrm{CI} \pm 1.3$ ) and itchy and watering eyes (IR 14.0, 95\% CI \pm 1.1 ) were always the most common complaints, although all of the symptoms, (with the exception of nausea) listed in the table were reported at each site. Most of the occupants registering complaints stated that their symptoms were a result of either entering or working in the building and most stated that the symptoms usually went away during weekends and holidays and returned on entering the building. More than half of the occupants that had IAQ complaints also complained of increased respiratory infections (such as tonsillitis, bronchitis, and some cases of pneumonia) (IR 14.3, 95\% $\mathrm{CI} \pm 1.0)$. Over one third of the occupants that registered complaints claimed that an increase in the relative humidity resulted in an increase in the severity of their symptoms (IR 12.0, 95\% $\mathrm{CI} \pm 0.9)$.

At most schools, before the IAQ company's investigations, public health departments had conducted investigations and used questionnaires. These investigations consisted primarily of measuring the $\mathrm{CO}_{2}$ in the buildings and increasing the ventilation rates, but with no success in reducing the IAQ complaints. The symptoms from the public health departments' questionnaires correlated with the symptoms obtained from the IAQ company's questionnaire. At all schools, many of the occupants that had IAQ complaints had submitted letters to the school officials, detailing their complaints and symptoms. The complaints and symptoms listed in these letters correlated with the complaints and symptoms obtained from the IAQ company's questionnaire.

After remedial action to any building, questionnaires were made available to the staff. They were asked to say if there were any complaints or symptoms related to the IAQ of the building. There were none or only a few complaints concerning the IAQ from the buildings that underwent remedial action. Complaints after the action were never registered by more than $3 \%$ of the staff at any school. The overall incidence was 2.5/100 employees (95\% CI $\pm 1.1)$. This represented a significant reduction $(p<0.001)$ in the number of IAQ complaints.

\section{CARBON DIOXIDE CHEMICAL AND PARTICULATE} MEASUREMENTS

Although $\mathrm{CO}_{2}$ concentrations were higher indoors than outdoors, there were no significant correlations between the indoor concentrations and the complaints or symptoms (data not shown). All measured constituents were well within the normal acceptable range of a school or office and there were no significant correlations between the indoor concentrations and complaints or symptoms (data not shown). No correlations were found between the outdoor particulate measurements, the indoor complaint areas, and the non-complaint areas. 


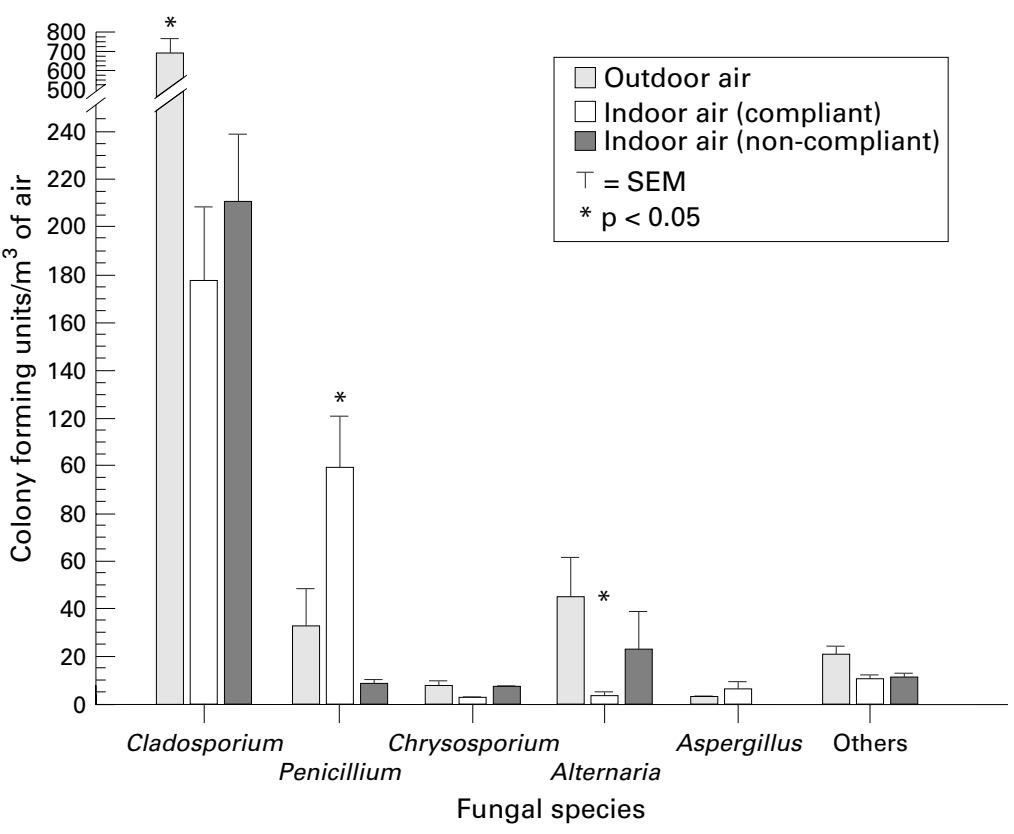

Figure 1 Bar graph of all air samples taken at the 48 schools.

Five fungal genera were consistently found in the outdoor air and comprised over $95 \%$ of the outdoor fungi (fig 1). These genera were Cladosporium (81.5\%), Penicillium (5.2\%), Chrysosporium (4.9\%), Alternaria (2.8\%), and Aspergillus (1.1\%). Other fungi (Fusarium, Epicoccum, Botrytis, Bipolaris, Acremonium, Drechslera, Rhizopus, Mucor, and Rhodotorula) were present in very low numbers and varied according to location and season. The predominant Cladosporium species isolated from the outdoor samples was Cladosporium cladosporioides. Several other Cladosporium species (such as Cladosporium herbarum and Cladosporium sphaerospermum) were also isolated, but these isolates were usually found in association with Cladosporium cladosporioides. The predominant Penicillium species isolated was Penicillium chrysogenum. Aspergillus niger was the most commonly isolated Aspergillus species from outdoor air samples. With the exception of a few sites along the northern Atlantic coast, most of the buildings were in mild temperate zones, with little or no snowfall, and an average relative humidity range of $30 \%-60 \%$. The rainfall in the survey areas was not abnormal, with the exception of states surrounding the southern region of the Gulf of Mexico, which were experiencing a drought during the survey period. The outdoor temperature was seasonal, varying from a low near $5^{\circ} \mathrm{C}$ to a high of $38^{\circ} \mathrm{C}$.

INITIAL INDOOR AIR SAMPLES

In most of the schools (fig 1), there were significant reductions in the $\mathrm{CFU} / \mathrm{m}^{3}$ of fungi in the indoor air samples from non-complaint areas compared with the outdoor air sample, but the fungal profiles were similar to outdoor air. In all of the buildings, the $\mathrm{CFU} / \mathrm{m}^{3}$ of Cladosporium species were significantly $(p<0.05)$ lower in the indoor air samples of non-complaint areas than in the outdoor air sample. In the indoor air sample complaint

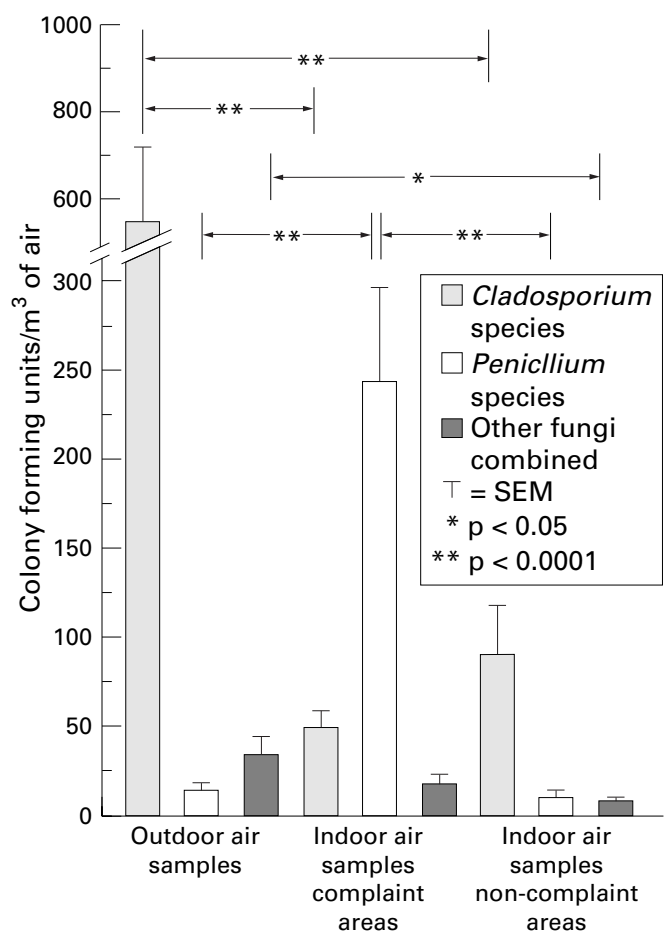

Figure 2 Bar graph of all air samples taken at the 20 schools where Penicillium species were the dominant fungi.

areas, the $\mathrm{CFU} / \mathrm{m}^{3}$ of Cladosporium species were lower, but not always significantly lower, than the outdoor air sample. Penicillium species and Aspergillus species were the only fungi isolated from the indoor air sample complaint areas that had higher $\mathrm{CFU} / \mathrm{m}^{3}$ of air when compared with the outdoor air sample and indoor air sample non-complaint areas. All of the schools had similar interior temperatures $\left(23^{\circ} \mathrm{C}\right)$. Most complaint sites showed very little HVAC maintenance as well as active water leaks.

At 20 schools (fig 2), there were significant increases $(\mathrm{p}<0.0001)$ in the $\mathrm{CFU} / \mathrm{m}^{3}$ of Penicillium species in the indoor air samples of complaint areas compared with the outdoor air sample and the indoor air samples of noncomplaint areas. The swab samples from these sites had very heavy growth of Penicillium species. The mean (SD, range) indoor relative humidity in these complaint areas (IRH-C) was $50 \%(12 \%, 23 \%-67 \%)$, in non-complaint areas (IRH-NC) $40 \%(10 \%, 30 \%-48 \%)$. The mean (SD, range) outdoor relative humidity (ORH) was $46 \%(20 \%, 22 \%-81 \%)$. Penicillium chrysogenum was the dominant fungal isolate in 14 of these sites.

In the air samples from complaint areas at five schools (data not shown) there were increases, although not significant $(p=0.10)$, in the number of $\mathrm{CFU} / \mathrm{m}^{3}$ of Penicillium species. In these the IRH-C had a mean (SD, range) of $64 \%(9 \%, 54 \%-70 \%)$, the IRH-NC $56 \%$ (3\%, $54 \%-58 \%)$. The outdoor relative humidity had a mean (SD, range) of $69 \%(15 \%, 60 \%-86 \%)$. The swab samples from these sites showed very heavy growth of Penicillium species and heavy growth of Cladosporium species, which indicated possible fungal growth in the interior and a potential IAQ problem. Penicillium chrysogenum was the most common isolate. 
At 11 schools (data not shown), Stachybotrys atra, which was not isolated from any air samples, was isolated from swabs of the visible growth taken from under wetted carpets, interior painted gypsum board walls, and especially behind vinyl coverings on gypsum board walls that had been wetted in indoor complaint areas. The indoor air samples from these complaint areas were not significantly different from the indoor air samples of the noncomplaint areas and had profiles that were similar to the outdoor air samples. The mean (SD, range) of these IRH-C samples was $62 \%$ (5\%, 58\%-66\%). The mean (SD, range) outdoor relative humidity was $83 \%(11 \%$, $65 \%-90 \%)$.

Three Aspergillus species (Aspergillus glaucus, Aspergillus versicolor, and Aspergillus flavus) were isolated from interior air samples and swab samples in association with the Penicillium species, but Aspergillus species (Aspergillus flavus) were dominant in complaint areas at only one school. The swab samples from the interior showed heavy to very heavy growth of Aspergillus species, along with medium to heavy growth of Cladosporium species and Penicillium species. The indoor relative humidity at this site was $65 \%$ and the outdoor relative humidity was $75 \%$.

In the remaining 11 schools, the fungal ratios and $\mathrm{CFU} / \mathrm{m}^{3}$ of air (outdoor and indoor air samples) were not significantly different. The swab samples from the interior of these sites showed heavy to very heavy growth of Cladosporium species or Penicillium species, which indicated possible fungal growth in the interior. The mean (SD, range) indoor relative humidity was $60 \%(3 \%, 56 \%-64 \%)$ and outdoors it was $60 \%(2 \%, 57 \%-62 \%)$.

INDOOR AIR SAMPLES AFTER REMEDIAL ACTION Indoor air samples and swab samples were retaken within 60 days of completion of the remedial action to a building and again at least 6 months after the action. At all sites the fungal ratios (outdoor air samples compared with indoor air samples) were very similar but the fungal $\mathrm{CFU} / \mathrm{m}^{3}$ from the indoor air samples were $50 \%-90 \%$ less $(p<0.05)$ than the outdoor air samples (data not shown). The indoor relative humidity had a mean (SD) of $44 \%(5 \%)$, with no site exceeding $57 \%$. The outdoor relative humidity ranged from $37 \%-87 \%$. All swab samples showed very light to light growth of Cladosporium species and a mixture of other species.

\section{Discussion}

All of the IAQ complaints investigated were generated by the occupants. As the validity of results from questionnaire studies may be altered by biases introduced by the observer or by the respondents, ${ }^{26}$ the results must be carefully weighed. Even when observer bias is reduced, the bias introduced by the respondent remains a potential source of systematic error. ${ }^{26}$ A major problem, however, is that it is often difficult to control independent variables because of the diversity of the study population, its motility, or a lack of personal exposure.
Most of the occupants (90\%) in the buildings that we investigated were teachers. Although potential psychological disorders-such as depression or anxiety-were not directly considered, we did, at most schools, find a high job satisfaction and a genuine concern for the welfare of the students. Also, it is difficult to segregate the individual pollutant from copollutants and other confounders. However, tobacco smoke can be eliminated as a potential confounder as all of the schools prohibited tobacco use on the campuses.

A causal relation is rarely discernible even with strong statistical significance. Thus, at best, associations can be drawn only between the exposure and the effect.

Although fungal contamination in indoor environments has been shown to produce allergies in occupants of these buildings, ${ }^{161827} 28$ the role of fungi in IAQ problems has become increasingly controversial. Our studies show that Penicillium species and Stachybotrys species are strongly associated with SBS. These data show that the Penicillium species, especially Penicillium chrysogenum, can adapt to an environment in which humans are most comfortable. Our studies also support earlier findings that Penicillium species has become an important indoor contaminant. ${ }^{29}$ This ubiquitous organism's optimal growth occurs between $10^{\circ}$ and $25^{\circ} \mathrm{C}$. It can grow over a wide range of water availability and has low water activity, although sporulation requires a higher water activity. ${ }^{30}$ Whereas it is widely stated that relative humidity $>70 \%$ is needed for active fungal growth, the water activity of the substrate is actually the critical variable. ${ }^{31}$ In the complaint areas where Penicillium species were dominant, we found (with the exception of the HVAC system at the fan during cooling) that the range of the indoor relative humidity was from $23 \%-67 \%$. Penicillium chrysogenum is apparently capable of successfully competing with most conidial fungi over almost the entire range of water availability. Its spores are small $(1-5 \mu \mathrm{m})$ and are capable of entering the lower respiratory tract. It has been shown that bronchial challenges with Penicillium species spores induced immediate and delayed type asthma in sensitised subjects. ${ }^{27}$

Stachybotrys species, some of which are capable of producing potent mycotoxins, ${ }^{32}$ require abnormally high relative humidity or wetted surfaces to grow. This fungus has been associated with illness related to buildings and SBS. ${ }^{33} 34$ It is difficult to isolate Stachybotrys species from the air and the presence of Stachybotrys may have been overlooked due to this phenomenon. Our findings suggest that when the fungal ecology of complaint air samples are similar to the outdoor air and non-complaint air samples, coupled with abnormally high interior relative humidity and symptoms of SBS, the possibility exists that a mycotoxin producing fungus such as Stachybotrys, may be hidden and growing in the interior of the building.

Spores of Cladosporium species probably occur more abundantly world wide than any 
other spore type and are the dominant airborne spores in many areas, especially in temperate climates. ${ }^{35}{ }^{36}$ Similar to other studies, we found Cladosporium cladosporioides growing inside buildings on various building materials. ${ }^{37} 38$ Although our findings showed that Cladosporium cladosporioides was not associated with the indoor air sample complaint areas, its presence indicated that the conditions favoured fungal growth that could potentially allow fungal genera such as Penicillium or Aspergillus to become the dominant organism.

The underlying factor for SBS is the modern, sealed building with its environment controlled by an HVAC system. These systems probably contribute to the onset of SBS by allowing build up of pollutants when the capacity of the HVAC system is inadequate or has been compromised. Our finding suggests that the initial stage of interior microbial growth began with water leaks that wetted various building materials. If these wetted materials are not properly mitigated, fungal growth may occur. Eventually, the HVAC system becomes contaminated. Although an understanding of the pollutants or conditions directly responsible for SBS is essential to developing strategies for prevention, a thorough analysis of the HVAC system, along with removing or properly repairing wetted areas, were often the key to mitigating the problem in a particular building.

After remedial action, the average air change per hour was 0.5. The air samples taken at 60 days and again at 6 months after remedial action had at least a $50 \%$ reduction in the number of $\mathrm{CFU} / \mathrm{m}^{3}$ from the indoor air sample than the outdoor air sample. For particles with an aerodynamic size $\geqslant 2.5 \mu \mathrm{m}$, the current evidence suggests that 1.0 air change per hour results in indoor concentrations of about $30 \%$ $80 \%$ of that outdoors. ${ }^{39}$

These findings show that remedial action to the buildings removed interior fungal growth. With a significant reduction in complaints about IAQ from occupants, our data suggest that Penicillium (Penicillium chrysogenum) species and Stachybotrys species may be strongly associated with SBS.

We gratefully acknowledge Curt White for early microbiological sample identification, and Ross Thompson and Damon Cooley for technical assistance. This work was supported by QIC Systems, Dallas, Texas and a Biomedical Research Grant from TTUHSC.

1 Hodgson M. Field studies on the sick building syndrome. Ann NY Acad Sci 1992;641:21-36.

2 Spangler JD, Sexton K. Indoor air pollution: a public health perspective. Science 1983;221:9-17.

Finnigan MS, Pickering CAC, Burge PS. The sick building syndrome: prevalence studies. BMF 1984;289:1573-5.

4 Mishra SK, Ajello L, Ahearn DG, et al. Environmental mycology and its importance to public health. F Med Vet Mycol 1992;30:287-305.

5 Feder G. Sick building syndrome. BMF 1985;290:322.

6 National Academy of Sciences. Indoor pollutants. Washington, DC: National Academy Press, 1981.

7 Sterling TD, Arundel A. Possible carcinogenic components of indoor air, combustion byproducts, formaldehyde, min- eral fibres, radiation, and tobacco smoke. $\mathcal{F}$ Environ Sci Health B 1984;2:185-230.

8 Bernstein RS, Sorenson WG, Garabrant D, et al. Exposures to respirable, airborne Penicillium from a contaminated ventilation system: clinical, environmental and epidemiological aspects. Am Ind Hyg Assoc $\mathcal{F}$ 1983;44:161-9.

9 Burge HA. Bioaerosols: prevalence and health effects in the indoor environment. F Allergy Clin Immunol 1990;86:687701.

10 Dales RE, Burnett R, Zwanenburg H. Adverse health effects among adults exposed to home dampness and molds. $A m$ Rev Respir Dis 1991;143:505-9.

11 Huuskonen MS, Husman K, Jarvisalo JJ, et al. Extrinsic allergic alveolitis in the tobacco industry. $\mathrm{Br} \mathcal{F}$ Ind $\mathrm{Med}$ 1984;41:77-83.

12 Solomon WR. Fungus aerosols arising from cold-mist vaporizers. F Allergy Clin Immunol 1974;54:222-8.

13 Solomon WR. Assessing fungus prevalence in domestic interiors. F Allergy Clin Immunol 1975;56:235-42.

14 Roby RR, Sneller MR. Incidence of fungal spores at the homes of allergic patients in an agricultural community. II. Correlation of skin test with mold frequency. Ann Allergy 1979;43:286-8.

15 Burrell R. Microbiological agents as health risks in indoor air. Environ Health Perspect 1991;95:29-34.

16 Lehrer SB, Aukrust L, Salvaggio JE. Respiratory allergy induced by fungi. Clin Chest Med 1983;4:23-41.

17 Miller ID. Fungi as contaminants of indoor air. Atmosphere and the Environment 1992;26:2163-72.

18 Salvaggio J, Aukrust L. Mold-induced asthma. F Allergy Clin Immunol 1981,68:327-46.

19 Wanner HU, Verhoeff AP, Colombi A, et al. Indoor air quality and its impact on man. Biological particles in indoor environments. Brussels, Luxembourg: Commission of the environments. Brussels, Luxembourg: Comm

20 Frey D, Oldfield RJ, Bridger RC. Color atlas of pathogenic fungi. Chicago: Year Book Medical Publishers, 1979.

21 Larone DH. Medically important fungi - a guide to identification, 2 nd ed. Washington, DC: American Society for Microbiology, 1993.

22 Rameriza C. Manual and atlas of the Penicillia. Amsterdam: Elsevier, 1982.

23 Samson RA, Pitt JI. Modern concepts in Penicillium and Aspergillus classification. New York: Plenum Press, 1990.

24 National Air Duct Cleaners Association. NADCA Standard 1992-01. Mechanical cleaning of non-porous air conveyance 1992-01. Mechanical cleaning of non-porous air conveya
system components. Washington, DC: NADCA, 1992.

25 Freund JE, Simen GA, eds. Modern elementary statistics, 8th ed. Englewood Cliffs, NJ; Prentice-Hill, 1992:287-9.

26 Samet JM. A historical and epidemiologic perspective on respiratory symptoms questionnaires. $\mathrm{Am} \mathcal{F}$ Epidemiol 1978;108:435-46.

27 Licorish K, Novey HS, Kozak P, et al. Role of Alternaria and Penicillium spores in the pathogenesis of asthma. $\mathcal{F}$ Allergy Clin Immunol 1985;76:819-25.

28 Verhoeff AP, van Strein RT, van Wijnen JH, et al. Damp housing and childhood respiratory symptoms: the role of sensitization to dust and molds. Am $\mathcal{F}$ Epidemiol 1998;141: 103-10.

29 Burge HA, Hoyer ME, Solomon WR. Quality control factors for Alternaria allergens. Mycotaxon 1989;34:55-63.

30 Pitt JI. Food spoilage and biodeterioration. In: Cole GT, Kendrick B, eds. Biology of conidial fungi. Vol 2. New York: Academic Press, 1981.

31 Gravesen S. Fungi as a cause of allergic disease. Allergy 1979;34:135-54.

32 Sorenson WG, Frazer DG, Jarvis BB, et al. Tricothecene mycotoxins in aerosolized conidia of Stachybotrysatra. Appl Environ Microbiol 1987;53:1320-5.

33 Update: Pulmonary hemorrhage or hemosiderosis among infants: Cleveland, Ohio, 1993-6. Morbidity and Mortality Weekly Report 1997:46:33-5.

34 Johanning E, Biagini R, Hull D, et al. Health and immunology study following exposure to toxigenic fungi (Stachybotrys chartarum) in a water-damaged office environment. Int Arch Occup Environ Health 1996:68:207-18.

35 Solomon WR, Matthews KP. Aerobiology and inhalant allergens. In: Middleton E, Reed CE, Ellis EF, et al, eds. Allergy: principles and practice, $3 r d$ ed. St Louis: Mosby, 1988:312-72

36 Lacey J. The aerobiology of conidial fungi. In: Cole GT Kendrick B, eds. Biology of conidial fungi. New York: Kendrick B, eds. Biology of com
Academic Press, 1981:373-416.

37 Ahearn DG, Price DL, Simmons RB, et al. Colonization studies of various HVAC insulation materials. In: $I A Q$ ' 92 Environment for People. Atlanta, GA: ASHRAE, 1992: 101-5.

38 Ahearn DG, Simmons RB, Switzer KF, et al. Colonization by Cladosporium species of painted metal surfaces associated with heating and air conditioning systems. Fournal of Industrial Microbiology 1991;8:277-80.

39 Costa DL, Amdur MO. Air pollution. In: Klaasen CD, ed Casarrett and Doull's toxicology: the basic science of poisons, 5th ed. New York: McGraw-Hill, 1996:857-82. 